\title{
FATORES DE RISCO PARA TENTATIVA DE SUICÍDIO: PRODUÇÃO DE CONHECIMENTO NO BRASIL
}

\section{RISK FACTORS FOR SUICIDE ATTEMPT: LITERATURE REVIEW}

\author{
Tamires Alexandre Félix, ${ }^{1}$ Eliany Nazaré Oliveira, ${ }^{1}$ Marcos Venicios de Oliveira Lopes, ${ }^{2}$ José Reginaldo Feijão \\ Parente, ${ }^{1}$ Maria Socorro de Araújo Dias, ${ }^{1}$ Roberta Magda Martins Moreira ${ }^{1}$ \\ ${ }^{1}$ Universidade Estadual Vale do Acaraú/Sobral/Brasil. ${ }^{2}$ Universidade Federal do Ceará-UFC/Fortaleza/Brasil. \\ Autor correspondente: Tamires Alexandre Félix e-mail: tamiresafelix @ gmail.com
}

\section{EDITORES}

Thiago Gomes Heck

(Unijuí-Brasil)

Adriane Cristina Bernat Kolankiewicz

(Unijuí-Brasil)

\section{EDITORES DE ÁREA}

Educação \& Saúde

Eva Teresinha de Oliveira Boff

(Unijuí-Brasil)

Fisioterapia \& Saúde

Eliane Roseli Winkelmann

(Unijuí-Brasil)

Ciências Farmacêuticas \& Saúde

Marilei Uecker Pletsch

(Unijuí-Brasil)

\section{Nutrição \& Saúde}

Lígia Beatriz Bento Franz

(Unijuí-Brasil)

Nadia Oliveira

(Unipampa-Brasil)

Ingrid Perry

(UNESC-Brasil)

\author{
Enfermagem e suas contribuições para \\ a prática \\ Adriane Cristina Kolankiewicz \\ (Unijuí-Brasil) \\ Crhis de Brum \\ (UFFS-Brasil) \\ Neila de Souza \\ (UFSM-Brasil) \\ Exercício Físico \& Saúde \\ Thiago Gomes Heck \\ (Unijuí-Brasil) \\ Anderson Zampier Ulbrich \\ (UFPR-Brasil)
}

Editora Unijuí

Universidade Regional do Noroeste do

Estado do Rio Grande do Sul (Unijuí)

\section{RESUMO}

O suicídio consiste num fenômeno único desde a motivação até o óbito, sendo a Tentativa de Suicídio (TS) o momento mais propício a intervenções em saúde. O artigo tem como objetivo evidenciar os fatores de risco para a TS revisando as produções publicadas no Brasil em 10 anos. Identificou-se prevalência de TS em mulheres, jovens, que vivem sozinhos, desempregados e com baixa escolaridade, fortemente relacionada a um contexto psicossocial de adoecimento e ao uso de drogas psicoativas. Entre os fatores de risco estão a falta de apoio social e espiritual, restrição de lazer e eventos estressantes. Os resultados indicam que a prevenção do suicídio deve ser mais valorizada do que o diagnóstico e o tratamento.

Palavras-chave: Risco. Tentativa de suicídio. Epidemiologia.

Submetido em: $21 / 6 / 2016$

Aceito em: 9/9/2016 


\section{Revista}

\section{Contexto}

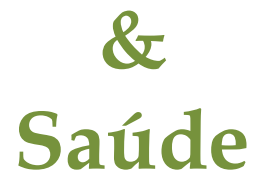

Volume 16

Número 31

2016

ISSN 2176-7114

\begin{abstract}
Suicide ranges from motivation to death; with the Suicide Attempt (AS) being the most propitious time for health interventions. Identify risk factors for SA, reviewing articles that address this issue in Brazil in 10 years. Showed prevalence of SA in women, young adults, that live alone, the unemployed and those with little schooling strongly associated to a psychosocial context of illness and the use of psychoactive drugs. Among the risk factors are lack of social and spiritual support, leisure restriction and occurrence of stressful events. The results indicate that suicide prevention needs to be more highly valued than situational diagnosis and treatment.
\end{abstract}

Keywords: Risk. Suicide. Attempted. Epidemiology.

\begin{abstract}
A Revista Contexto \& Saúde é um periódico do Departamento de Ciências da Vida da Universidade Regional do Noroeste do Estado do Rio Grande do Sul (Unijuí). É um periódico semestral que tem por objetivo a divulgação da produção técnico-científica de temas relacionados à área de Ciências da Saúde.

O escopo da revista abrange a divulgação de resultados de pesquisa que contemplem avanços no processo saúde-doençacuidado e no conhecimento e aplicabilidade de novos processos químicos e biológicos em saúde.

Neste periódico, entende-se que a publicação de estudos com os aspectos epidemiológicos, assistenciais e educacionais em saúde, experimentais e aplicados é uma forma a subsidiar e qualificar a atenção à saúde de modo interdisciplinar.
\end{abstract}




\section{INTRODUÇÃO}

O suicídio é um fenômeno humano complexo, universal, e representa um grande problema de saúde pública em todo o mundo (VIDAL; GONTIJO, 2013). Pode-se definir o suicídio como a morte de uma pessoa causada por autoagressão, no entanto esse fenômeno inclui desde a motivação e ideação de autoextermínio até o planejamento do ato, a tentativa e o óbito (ABREU et al., 2010).

Nesse percurso, que envolve dimensões subjetivas de difícil avaliação, diagnóstico e monitoramento, pode-se afirmar que a tentativa de suicídio (TS) é o momento mais propício a intervenções em saúde, demandando maior investigação com a finalidade de aprimorar o cuidado e prevenir a violência autodirigida.

As TSs podem ser conceituadas como atos intencionais de autoagressão que não resultam em morte (SOUZA et al., 2011). Atualmente, no Brasil, tais tentativas são consideradas agravos de notificação compulsória (BRASIL, 2007; BARROS, 2011) e envolvem aspectos culturais, mitos, tabus, estigmas e fatores biopsicossociais pouco discutidos na literatura, apesar da progressão das taxas de suicídio em âmbito nacional.

A TS é um importante preditor para suicídio e tem gerado encargos econômicos e familiares decorrentes de lesões incapacitantes e do adoecimento do núcleo de convívio da pessoa que tentou suicidar-se. Pesquisas mostram que cada ato suicida afeta profundamente e por tempo prolongado pelo menos cinco pessoas próximas (TARANTINO, 2007).

Em todo o mundo, cerca de 800 mil pessoas cometem suicídio todos os anos, o que corresponde a uma taxa de 11,4 óbitos para cada 100 mil habitantes e 1 morte autoprovocada a cada 40 segundos. Estima-se que o número de TSs supere esse índice em até 10 vezes (WHO, 2014).

O Brasil está classificado como oitavo país em números absolutos correspondentes a esse agravo, entretanto as Diretrizes Nacionais para Prevenção do Suicídio (BRASIL, 2006) e o Plano Nacional de Prevenção do Suicídio ainda têm aplicabilidade limitada, devido à dificuldade de identificar essa demanda para estabelecer a produção do cuidado como preconizado.

O modelo biomédico determina a TS como consequência de um transtorno psiquiátrico de gênese biológica, desconsiderando aspectos transversais à vida humana que também interferem na saúde mental, como condições socioeconômicas, estrutura familiar, eventos estressantes, padrões culturais, consumo de drogas, entre outros que interagem com as predisposições biológicas para o surgimento do comportamento suicida.

Já na perspectiva do modelo organizacional em redes de atenção, os profissionais da saúde devem estar aptos a detectar precocemente o comportamento suicida e a desenvolver projetos terapêuticos a grupos de risco e de pessoas que tentaram o suicídio e sobreviveram, desenvolvendo ações que envolvam a família com o objetivo de prevenir danos e promover reabilitação psicossocial.

Nesse contexto, a identificação de grupos de risco de TS e o acompanhamento dos sobreviventes são componentes-chave para o enfrentamento do problema e para a gestão do cuidado em saúde mental (SOUZA et al., 2011).

Este artigo busca evidenciar os fatores de risco de TS ao revisar as produções que analisam essa questão no Brasil, com o objetivo de sistematizar os achados da literatura para subsidiar ações de prevenção intersetoriais mais eficazes. 


\section{METODOLOGIA}

Este estudo consiste em uma revisão integrativa da literatura, considerada uma abordagem metodológica ampla que fundamenta a prática baseada em evidências a partir da sistematização dos achados mais consistentes sobre determinado tema.

Dada a relevância da revisão integrativa, como técnica para sintetizar o conhecimento aplicado à saúde promovendo estratégias de cuidado mais eficazes, utilizamos como referencial metodológico Souza, Silva e Carvalho (2010). Estes autores determinam seis etapas no processo de elaboração da revisão, a saber: elaboração da pergunta norteadora; - busca ou amostragem na literatura; - coleta de dados; - análise crítica dos estudos incluídos; - discussão dos resultados; - apresentação da revisão integrativa. Assim, garante-se a fidedignidade dos dados, permitindo uma análise aprimorada do assunto, das lacunas de pesquisa e possibilidades de intervenção.

As questões norteadoras desta pesquisa foram: "Quais fatores de risco se associam à tentativa de suicídio no Brasil?”; “Que conhecimento tem sido produzido no país sobre risco de tentativa de suicídio?”; “Quais são os principais achados relacionados ao tema?". Empreendeu-se a coleta nas bases de dados Literatura LatinoAmericana e do Caribe em Ciências da Saúde (Lilacs), Scientific Electronic Library Online (Scielo) e Medline (Sistema On-Line de Busca e Análise de Literatura Médica), fontes abrangentes de publicações científicas em saúde pública.

A busca foi realizada em agosto de 2015 a partir do cruzamento dos descritores na seguinte combinação: "tentativa de suicídio" e "risco" ou "fatores de risco". Os critérios de inclusão foram: artigo científico publicado entre 2005 e 2015, disponível para acesso gratuito sem restrição de idiomas de publicação. Os critérios de exclusão foram: estudos que não se relacionavam à realidade brasileira e produções repetidas nas bases de dados.

Vale ressaltar que algumas pesquisas apresentavam dados relacionados ao risco de TS em pacientes com patologias ou condições clínicas específicas, como portadores do vírus HIV, puérperas, pacientes oncológicos, obesos e gestantes de alto risco, apontando a doença de base como o maior fator de risco. Compreendeu-se que esses estudos não atendem à proposta por não relacionar fatores gerais que possam afetar as populações com ideação suicida, sendo incluídos como literatura complementar.

Quadro 1 - Apresentação da amostragem na literatura incluída na revisão integrativa após aplicação dos critérios de inclusão e exclusão.

\begin{tabular}{|l|l|l|l|l|l|l|l|l|}
\hline & Total & $\begin{array}{c}\text { Disponíveis } \\
\text { para } \\
\text { acesso }\end{array}$ & Artigos & Brasil & $\begin{array}{c}\mathbf{2 0 0 5}- \\
\mathbf{2 0 1 5}\end{array}$ & $\begin{array}{c}\text { Correspondência } \\
\text { com os objetivos } \\
\text { da pesquisa }\end{array}$ & $\begin{array}{c}\text { Duplicados } \\
\text { nas bases } \\
\text { de dados }\end{array}$ & Final \\
\hline Lilacs & 618 & 294 & 231 & 47 & 41 & 12 & 1 & 11 \\
\hline Scielo & 102 & 93 & 91 & 82 & 75 & 8 & 1 & 7 \\
\hline Medline & 16.151 & 4.888 & 4.888 & 40 & 11 & 10 & 4 & 6 \\
\hline Amostra & 16.871 & 5275 & 362 & 169 & 127 & 30 & 6 & 24 \\
\hline
\end{tabular}

Fonte: Elaborado pelos autores.

Das pesquisas encontradas foram extraídas as seguintes informações: título, objetivo, ano de publicação, método, região na qual foi realizado o estudo e principais resultados. Elaborou-se um quadro sinóptico como 
instrumento de consulta conforme recomenda o referencial adotado, diminuindo o risco de erros e garantindo precisão na checagem das informações.

Após leitura crítica dos estudos na íntegra ponderando sobre o rigor e a característica de cada trabalho procedeu-se ao fichamento das evidências de forma hierárquica, dependendo da abordagem metodológica utilizada. A seguir apresentam-se os resultados.

\section{RESULTADOS}

Todos os artigos incluídos foram lidos na íntegra e agrupados por similaridade de conteúdo, constituindo três categorias finais, apresentadas e discutidas a seguir, que enfatizam os objetivos e as principais contribuições das pesquisas. na sequência apresenta-se quadro sinóptico (Quadro 2) com informações resumidas sobre as produções incluídas na pesquisa.

Quadro 2 - Distribuição dos estudos incluídos na revisão integrativa

\begin{tabular}{|c|c|c|c|}
\hline Título & Ano & Região & Método \\
\hline \multicolumn{4}{|l|}{ Base de Dados Lilacs } \\
\hline $\begin{array}{l}\text { Perfil das tentativas de suicídio por sobredose } \\
\text { intencional de medicamentos atendidas por um Centro } \\
\text { de Controle de Intoxicações do Paraná, Brasil. }\end{array}$ & 2010 & Sul & $\begin{array}{l}\text { Estudo } \\
\text { retrospectivo } \\
\text { descritivo. }\end{array}$ \\
\hline $\begin{array}{l}\text { The influence of the comorbidity between depression } \\
\text { and alcohol use disorder on suicidal behaviors in the } \\
\text { São Paulo Epidemiologic Catchment Area Study, } \\
\text { Brazil. }\end{array}$ & 2010 & Sudeste & $\begin{array}{l}\text { Estudo } \\
\text { transversal. }\end{array}$ \\
\hline $\begin{array}{l}\text { Suicide attempts and substance use in an emergency } \\
\text { room sample. }\end{array}$ & 2009 & Sudeste & $\begin{array}{l}\text { Estudo } \\
\text { epidemiológico } \\
\text { descritivo. }\end{array}$ \\
\hline $\begin{array}{l}\text { Suicide risk among inpatients at a university general } \\
\text { hospital. }\end{array}$ & 2007 & Sudeste & Quantitativo. \\
\hline $\begin{array}{l}\text { Apoio social, eventos estressantes e depressão em casos } \\
\text { de tentativa de suicídio: um estudo de caso-controle } \\
\text { realizado em um hospital de emergência do Rio de } \\
\text { Janeiro. }\end{array}$ & 2010 & Sudeste & $\begin{array}{l}\text { Estudo caso- } \\
\text { controle. }\end{array}$ \\
\hline $\begin{array}{l}\text { Polifarmácia e tentativas de suicídio no transtorno } \\
\text { bipolar. }\end{array}$ & 2007 & Sudeste & $\begin{array}{l}\text { Estudo } \\
\text { transversal. }\end{array}$ \\
\hline $\begin{array}{l}\text { Bipolar disorder first episode and suicidal behavior: are } \\
\text { there differences according to type of suicide attempt? }\end{array}$ & 2009 & Sudeste & Quantitativo. \\
\hline $\begin{array}{l}\text { Tentativas de suicídio atendidas em unidades públicas } \\
\text { de saúde de Fortaleza-Ceará, Brasil. }\end{array}$ & 2014 & Nordeste & Quantitativo. \\
\hline $\begin{array}{l}\text { Prevalência de transtornos mentais nas tentativas de } \\
\text { suicídio em um hospital de emergência no Rio de } \\
\text { Janeiro, Brasil. }\end{array}$ & 2009 & Sudeste & Quantitativo. \\
\hline $\begin{array}{l}\text { Gender differences in suicide attempts: preliminary } \\
\text { results of the multisite intervention study on suicidal } \\
\text { behavior (Supre-Miss) from Campinas, Brazil. }\end{array}$ & 2007 & Sudeste & $\begin{array}{l}\text { Estudo } \\
\text { transversal não } \\
\text { controlado. }\end{array}$ \\
\hline Tentativas de suicídio em um hospital geral no Rio de & 2006 & Sudeste & Estudo de \\
\hline
\end{tabular}




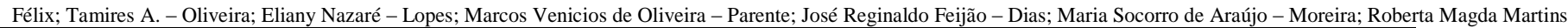
Janeiro, Brasil.

casos.

\section{Portal Scielo}

Ideação suicida e tentativas de suicídio no transtorno afetivo bipolar tipo I: uma atualização para o clínico.

Estudo de ideação suicida em adolescentes de 15 a 19 anos.

Tentativas de suicídio em jovens: aspectos epidemiológicos dos casos atendidos no setor de urgências psiquiátricas de um hospital geral universitário entre 1988 e 2004.

Tentativas de suicídio: uma análise de contingências.

Tentativa de suicídio entre pacientes com uso nocivo de bebidas alcoólicas internados em hospital geral.

Stressors in attempted suicide by poisoning: a sex comparison.

Fatores de risco para tentativa de suicídio por envenenamento: revisão.

\begin{tabular}{|c|c|c|}
\hline 2009 & Sudeste & $\begin{array}{l}\text { Revisão de } \\
\text { literatura. }\end{array}$ \\
\hline 2006 & Sul & $\begin{array}{c}\text { Estudo } \\
\text { transversal. }\end{array}$ \\
\hline 2008 & Sudeste & $\begin{array}{c}\text { Estudo de } \\
\text { casos. }\end{array}$ \\
\hline 2013 & Sul & Qualitativo. \\
\hline 2010 & Sudeste & $\begin{array}{c}\text { Estudo } \\
\text { transversal. }\end{array}$ \\
\hline 2012 & Nordeste & $\begin{array}{c}\text { Estudo } \\
\text { transversal e } \\
\text { comparativo. }\end{array}$ \\
\hline 2014 & Brasil & $\begin{array}{l}\text { Revisão } \\
\text { integrativa da } \\
\text { literatura. }\end{array}$ \\
\hline
\end{tabular}

\section{Medline}

Thinking about dying and trying and intending to die: results on suicidal behavior from a large Web-based sample.

Mood disorders hospitalizations, suicide attempts, and suicide mortality among agricultural workers and residents in an area with intensive use of pesticides in Brazil.

The impact of comorbid posttraumatic stress disorder on bipolar disorder patients.

Fatores associados à ideação suicida na comunidade: um estudo de caso-controle. Suicidal behavior in the community: prevalence and factors associated with suicidal ideation.

Uso de agrotóxicos e suicídios no Estado do Mato Grosso do Sul, Brasil.

\begin{tabular}{|c|c|c|}
\hline 2015 & Brasil & $\begin{array}{c}\text { Estudo } \\
\text { epidemiológico } \\
\text { descritivo. }\end{array}$ \\
\hline 2010 & Sudeste & Comparativo. \\
\hline 2010 & Brasil & Transversal. \\
\hline 2006 & Sudeste & $\begin{array}{c}\text { Estudo caso- } \\
\text { controle. } \\
\text { Estudo } \\
\text { epidemiológico } \\
\text { descritivo. } \\
\text { Estudo } \\
\text { epidemiológico } \\
\text { descritivo. }\end{array}$ \\
\hline 2005 & Sul \\
\hline
\end{tabular}

Fonte: Elaborado pelos autores. 
Gráfico 1 - Número de produções por ano dos estudos incluídos na revisão integrativa

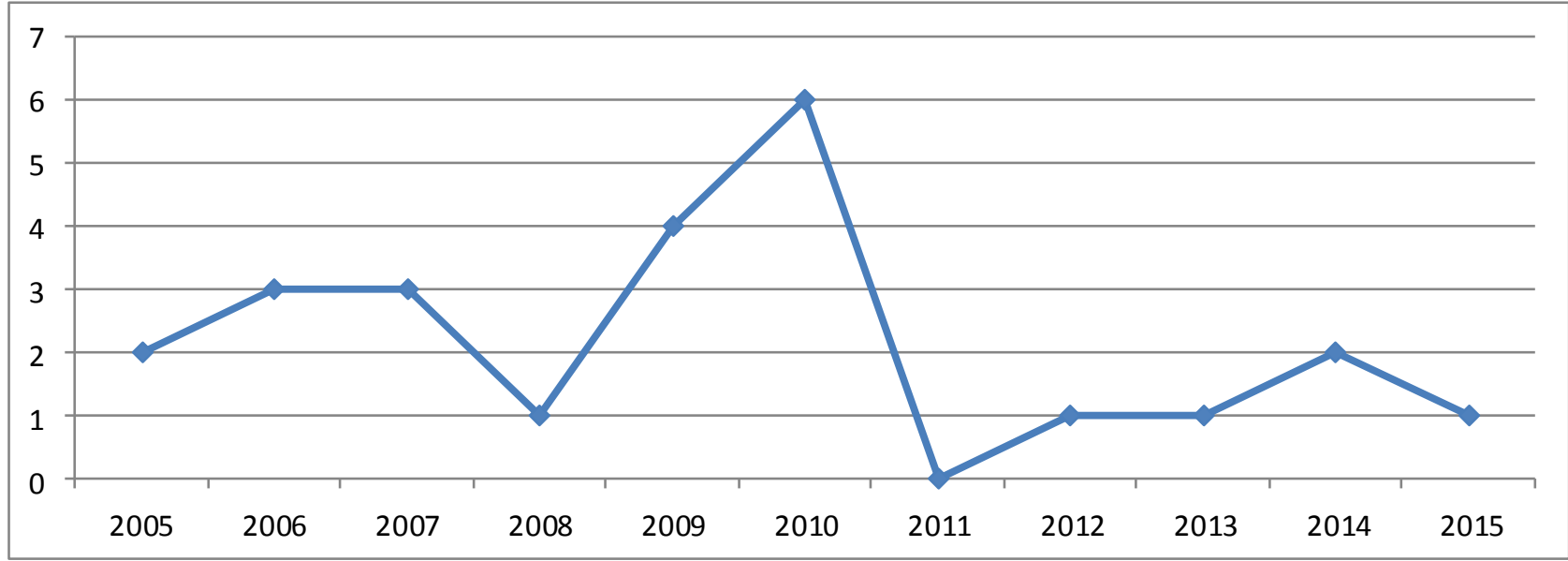

Fonte: Elaborado pelos autores.

Dos 24 artigos incluídos, 15 tratam da Região Sudeste. Este predomínio pode estar associado ao desenvolvimento ramificado do projeto Supre-Miss (Estudo Multicêntrico de Intervenção no Comportamento Suicida) da OMS na cidade de Campinas - São Paulo desde o ano de 2002.

\section{ASPECTOS SÓCIO-DEMOGRAFICOS DE RISCO PARA TENTATIVA DE SUICÍDIO}

A TS pode estar relacionada a fatores sociodemográficos diversos, que mudam segundo o contexto cultural, histórico e político. A maioria dos estudos abordados, no entanto, identificou maior prevalência de TS em mulheres, adolescentes e jovens, pessoas que vivem sozinhas, desempregados e indivíduos com baixa escolaridade. Considerando que são informações clinicamente úteis, que podem ajudar a identificar casos de risco nas populações para melhor acompanhamento e avaliação, maior compreensão sobre o tema mostra-se necessária.

A maioria dos artigos converge para a predominância da mulher na TS (BERNARDES; TURINI; MATSUO, 2010; SANTOS et al., 2009; PIRES et al., 2012; DIEHL; LARANJEIRA, 2009; STEFANELLO et al., 2008). Isso relaciona-se ao fato de que os homens demoram a buscar auxílio médico, encontrando-se, assim, com quadros mais graves quando chegam ao hospital após autoagressão (SANTOS et al., 2009). Esse achado também revela que o gênero define diferentes apresentações da lesão autoinfligida, mais especificamente nas motivações para o ato, no método utilizado (PIRES et al., 2012; DIEHL; LARANJEIRA, 2009; STEFANELLO et al., 2008) e nos transtornos mentais de base (FICHER; VANSAN, 2008).

Quanto à faixa etária, adolescentes e adultos jovens são os que mais tentam suicídio (BERNARDES; TURINI; MATSUO, 2010; SANTOS et al., 2009). Um estudo realizado com 526 adolescentes entre 15 e 19 anos, residentes em Porto Alegre, revelou que 36\% apresentavam ideação suicida (BORGES; WERLANG, 2006), índice elevado considerando a série temporal abordada. Outro estudo, cujo objetivo foi estimar a proporção de pacientes internados em um hospital geral universitário que se encontravam em risco de cometer suicídio, revelou que, em uma amostra aleatória de 253 pacientes, 5\% apresentavam alto risco de TS, sendo essa estimativa duas vezes maior para adultos jovens com menos de 30 anos (FERREIRA et al., 2007). 
Félix; Tamires A. - Oliveira; Eliany Nazaré - Lopes; Marcos Venicios de Oliveira - Parente; José Reginaldo Feijão - Dias; Maria Socorro de Araújo - Moreira; Roberta Magda Martins A literatura também aponta que o desemprego e o baixo nível socioeconômico incrementam o risco de TS entre indivíduos vulneráveis, como aqueles com história de TS (SANTOS et al., 2009). Assim, pessoas com baixa instrução educacional e sem ocupação, incluindo as "donas de casa" e os aposentados, tendem a desenvolver o percurso de autoagressão com maior facilidade e mais rapidamente devido a sua reduzida compreensão acerca do tratamento ou a sentimentos de culpa e inferioridade.

Um estudo nacional conduzido com 48.569 voluntários recrutados na Internet traçou um perfil semelhante ao descrito nos demais estudos evidenciando que o risco pode perpassar por uma série de variáveis comuns, o que direciona as intervenções em saúde voltadas para a população (ARAÚJO et. al., 2015). Silva et. al. (2006), no entanto, afirmam que não há distinção quanto aos fatores sociodemográficos para a ideação suicida, ou seja, desde o início do percurso não exercem potencial de risco para a violência autodirigida e sim, os sintomas depressivos e comorbidades psiquiátricas. Outros estudos sugerem que os mesmos fatores de risco tendem a operar distintamente nas diferentes etapas do suicídio.

A TS é um importante problema de saúde pública que precisa ser mais bem investigado por meio de metodologias adequadas à nossa realidade. Botega et. al. (2005) identificaram em uma amostra de 515 indivíduos uma prevalência de $17,1 \%$ para ideação suicida, 4,8\% para os planos e 2,8\% para tentativas revelando a magnitude da questão.

\section{CONTEXTO PSICOSSOCIAL: TRANSTORNOS PSIQUIÁTRICOS DE BASE E A TENTATIVA DE SUICÍDIO}

A TS está fortemente relacionada a um contexto psicossocial de adoecimento anterior, o que inclui convívio com pessoas depressivas ou que já tentaram suicídio, comorbidades psiquiátricas e uso de crack, álcool e outras drogas psicoativas (FONSECA et al., 2010).

Alguns autores afirmam que, entre todos os transtornos psiquiátricos, a depressão é a que se associa ao suicídio com maior frequência, seja como diagnóstico ou como sintoma (SANTOS et al., 2009; PIRES et al., 2012; DIEHL; LARANJEIRA, 2009; STEFANELLO et al., 2008; FICHER; VANSAN, 2008; BORGES, WERLANG, 2006). A depressão compõe a corrente do que se denomina suicídio contagioso. Pode-se afirmar que o convívio mórbido, conversar com pessoas que têm ideação de morte, transfere, de certa forma, uma profunda tristeza e reflexão sobre a vida que pode induzir outras pessoas já propensas a se automutilar.

Por exemplo, um estudo caso-controle identificou três vezes mais episódios depressivos (FONSECA et al., 2010) nos casos do que nos controles. Outra pesquisa ressaltou que, entre os 188 adolescentes com ideação suicida participantes, $100 \%$ conheciam alguém que havia tentado suicídio e 64,4\% conheciam alguém que se suicidou (BORGES; WERLANG, 2006).

Além da depressão, o transtorno bipolar de base tem sido referido na literatura como fator de risco de autoagressão, principalmente se o primeiro episódio foi de depressão e não de mania. Em pacientes com ciclagem rápida, a tendência é que em episódios posteriores esses indivíduos tentem o suicídio de maneira mais violenta, a depender das perspectivas genéticas, biológicas e do tratamento (NEVES et al., 2009).

Também foram identificados transtorno de estresse pós-traumático, transtornos de ansiedade e de personalidade, esquizofrenia, fobias e psicoses diversas em pessoas com história de TS (SANTOS et al., 2009; PIRES et al., 2012; DIEHL; LARANJEIRA, 2009; STEFANELLO et al., 2008; FICHER; VANSAN, 2008). Um artigo apresentou a prevalência de transtornos mentais em 71,9\% de uma amostra com 96 casos de TS, com divergência na prevalência dessas morbidades em relação à variável sexo (SANTOS et al., 2009). 
Outros artigos apresentam dados que indicam TS anterior, histórico familiar de autoagressão e depressão, algum tipo de deficiência física e o uso de medicamentos psicoativos como preditores de risco suicida, inclusive por intoxicação exógena devido a overdose dos próprios psicotrópicos (SANTOS et al., 2009; FICHER; VANSAN, 2008; FONSECA et al., 2010; WERNECK et al., 2006). Por exemplo, uma pesquisa apresentou a polifarmácia no transtorno bipolar como fator de risco de TS. Dos 169 pacientes incluídos, cinco em cada seis indivíduos (84\%) faziam uso de mais de uma medicação e $19 \%$ estavam usando mais de três drogas. Cerca da metade tinha história de TS (GAZALLE et al., 2007).

Embora os dados sobre suicídio considerem distúrbios isolados, em geral desordens psiquiátricas tendem a ser comorbidade e casos que satisfazem os critérios de uma única psicopatologia são raros (COÊLHO et al., 2010).

No que respeita ao uso de substâncias psicoativas e à determinação da violência autoinfligida, alguns estudos nacionais realizados em diferentes cenários demonstraram uma ampla variedade de prevalência, corroborando a existência de uma associação (DIEHL; LARANJEIRA, 2009; LIMA et al., 2010).

Um estudo realizado com 80 pessoas que tentaram suicídio identificou prevalência de $28,7 \%$ de consumo de drogas nas 6 horas anteriores à TS. A dependência da substância foi identificada em 10\% da amostra e, destes, todos já tinham história de TS (DIEHL; LARANJEIRA, 2009).

Um histórico de violência/abuso de ordem física, psicológica ou sexual, principalmente na infância, também está associado a maiores taxas de transtornos de humor e comportamento suicida na idade adulta. Isso também pode ser associado a níveis mais altos de impulsividade e maiores taxas de comorbidade com transtorno de personalidade, ambos fatores de risco de comportamento suicida (ABREU et al., 2009; FIGEL, MENEGATTIL; PINHEIRO, 2013).

Assim, torna-se evidente que o contexto psicossocial é determinante para prevenção ou estímulo do comportamento suicida. No Brasil faz-se necessário desenvolver pesquisas que avaliem os transtornos mentais relacionados às TSs, com a utilização de dados primários coletados por meio de instrumentos padronizados e validados internacionalmente, capazes de diagnosticar um grande número de transtornos mentais, possibilitando, assim, uma comparação com a literatura internacional (SANTOS et al., 2009).

\section{FATORES DE RISCO E VARIÁVEIS CLÍNICAS ASSOCIADAS À AUTOAGRESSÃO}

Uma vez que alguns indivíduos chegam a consumar o suicídio, as consequências desse ato envolvem um tratamento complexo para a família. Grande empenho tem sido dedicado para identificar fatores de risco de tal comportamento, visando a desenvolver ações de prevenção mais efetivas (BORGES; WERLANG, 2006).

Os fatores de risco mais comuns de TS apresentados nos estudos incluem frágil estrutura familiar, antecedentes psiquiátricos, individuais ou familiares, falta de apoio social, restrição de lazer, falta de apoio espiritual, consumo de drogas recreativas, conflitos conjugais, baixa escolaridade e eventos estressantes, sempre associados ao sexo e à intensidade da ideação suicida.

Uma pesquisa que abordou dez histórias clínicas de casos de TS em pacientes adolescentes revelou que a maioria deles era proveniente de lares desestruturados, predominantemente por separação dos pais, e que a tentativa ocorreu com maior frequência após discussão com pessoas importantes no núcleo sociofamiliar (FICHER; VANSAN, 2008). 
Félix; Tamires A. - Oliveira; Eliany Nazaré - Lopes; Marcos Venicios de Oliveira - Parente; José Reginaldo Feijão - Dias; Maria Socorro de Araújo - Moreira; Roberta Magda Martins Quanto aos eventos estressantes, estes podem estar relacionados à ausência de apoio social. Estudos têm demonstrado os efeitos deletérios da ausência de suporte social em situações estressantes, como a morte de pessoas íntimas, separação conjugal, desemprego ou qualquer mudança nociva no ambiente (FONSECA et al., 2010).

O Transtorno de Estresse Pós-Traumático vem se revelando importante preditor de autoagressão e marcador de prognósticos ruins. Quarantini et al. (2010) avaliaram pacientes com transtorno bipolar associado a Transtorno de Estresse Pós-Traumático identificando maior risco proporcional de tentativa de suicídio, alterações de humor e pior qualidade vida.

Um estudo de revisão apresentou os fatores de risco mais prevalentes em TS por envenenamento, método que hoje está entre os três mais comuns em casos de autoagressão. Os resultados indicaram que o transtorno psiquiátrico de base é o fator de risco mais relevante nesses episódios (PIRES et al., 2014). Pouco se sabe sobre como esses fatores de risco interagem no comportamento suicida, bem como sobre o contexto em que os indivíduos estão inseridos (FIGEL; MENEGATTIL; PINHEIRO, 2013; OLIVEIRA; BEZERRA FILHO; FEITOSA, 2014).

Em relação ao método utilizado, envenenamento ou intoxicação intencional é o mais citado nos estudos e o mais prevalente nas amostras de pesquisa (FICHER; VANSAN, 2008). A substância utilizada varia segundo o sexo, sendo a overdose medicamentosa mais comum entre mulheres e a ingestão de pesticidas e outras substâncias químicas de maior letalidade entre homens (BERNARDES; TURINI; MATSUO, 2010; FICHER; VANSAN, 2008; FONSECA et al., 2010; WERNECK et al., 2006).

Tendo em vista a prevalência de overdose intencional de medicamentos, os prescritores devem avaliar com maior cautela o estado psíquico e emocional do paciente antes de receitar medicamentos psicoativos, grupo farmacológico mais frequente e com maior risco toxicológico nessa situação (BERNARDES; TURINI; MATSUO, 2010; WERNECK et al., 2006).

Quanto à ingestão de pesticidas como método de autoextermínio, o estudo conduzido por Meyer et al. (2010) demonstrou que residir em áreas rurais pode elevar o risco, disparando a reflexão sobre a comercialização não regulada destes compostos, o armazenamento nas residências com grupos familiares de risco e a necessidade de educação em saúde nas comunidades rurais sobre o assunto.

Pires, Caldas e Recena (2005) estudaram os dados relativos à década de 1992-2002 numa área rural do Mato Grosso do Sul/Brasil. Durante o período foram notificados 1.355 casos de envenenamento por pesticidas, incluindo 506 tentativas de suicídio, resultando em 139 mortes. "O maior potencial de exposição a agrotóxicos dos trabalhadores rurais, nas pequenas propriedades da microrregião de Dourados, pode explicar o alto número de notificações de tentativa de suicídios e óbitos devido ao uso desses produtos” (p. 603).

Clinicamente, a natureza transitória dos sintomas, a eficácia da assistência hospitalar e a baixa letalidade do método contribuem para a alta precoce desses pacientes, diminuindo as chances de encaminhamento e seguimento do caso. Algumas intercorrências comuns decorrentes da hospitalização, no entanto, como a pneumonite, podem prolongar o tempo de internação e piorar o prognóstico (BERNARDES; TURINI; MATSUO, 2010).

Para efeitos de revisão da literatura, os estudos convergem em seus resultados, porém ainda carecem de abordagens qualitativas que indiquem os aspectos subjetivos e de cuidado na rede de atenção à saúde mental, principalmente na Atenção Primária à Saúde. 


\section{CONSIDERAÇÕES FINAIS}

Estudar as TSs é um desafio, pois isso envolve recursos tecnológicos, financeiros e humanos, constante atualização segundo a literatura, abordagem multissetorial e interprofissional, instrumentos válidos e sensibilidade, entretanto o impacto social é imenso em termos de perda de vidas, contexto de adoecimento e encargo familiar.

Consideramos que o número de estudos identificados foi reduzido, limitando-se aos fatores de risco mais prevalentes ou que se associam a um método específico de autoextermínio, e eles adotam abordagem quantitativa. Sugere-se, assim, o desenvolvimento de novas pesquisas no campo da determinação subjetiva dos casos, das histórias de vida, das condições clínicas e do diagnóstico dos transtornos psiquiátricos de base. Dessa forma, pode-se determinar os entraves que distanciam a realidade da pesquisa daquela observada nas populações em que as taxas de TS vêm crescendo progressivamente.

Há necessidade de investigar em maior profundidade as distinções de gênero e os aspectos culturais, sociais e regionais que influenciam o comportamento suicida nas diferentes comunidades. Os resultados também sugerem que a prevenção do suicídio precisa ser mais valorizada do que o diagnóstico situacional e o tratamento.

O risco de suicídio, como se observou, é de difícil determinação. Pesquisas que relatem experiências exitosas devem apresentar abrangência nacional, tendo em vista que 15 das 18 produções tratavam somente das regiões Sul ou Sudeste.

A série temporal abordada possibilitou incluir os estudos mais recentes, sintetizando o perfil das publicações, porém as limitações metodológicas apontam para a necessidade de estudos com escopo mais amplo publicados em forma de artigo.

\section{REFERÊNCIAS}

ABREU, K. P. et al. Comportamento suicida: fatores de risco e intervenções preventivas. Revista Eletrônica de Enfermagem, v. 12, n. 1, p. 195-200, 2010. Disponível em: <https://www.fen.ufg.br/fen_revista/v12/n1/pdf/v12n1a24.pdf>. Acesso em: 9 dez. 2015.

ABREU, L. N. et al. Suicidal ideation and suicide attempts in bipolar disorder type I: an update for the clinician. Revista Brasileira de Psiquiatria, São Paulo, v. 31, n. 3, p. 271-280, abr. 2009. Disponível em: <http://www.scielo.br/pdf/rbp/v31n3/aop0309.pdf >. Acesso em: 9 dez. 2015.

ARAÚJO, R. M et al. Thinking about dying and trying and intending to die: results on suicidal behavior from a large Web-based sample. J Clin Psychiatry, v. 76, n. 3, p. 359-365, mar. 2015.

BARROS, D. M. Notificação compulsória de violência: implicações em saúde mental. Revista de Psiquiatria Clínica, São Paulo, v. 38, n. 4, p. 125, 2011. Disponível em: 〈http://www.scielo.br/pdf/rpc/v38n4/a01v38n4.pdf〉. Acesso em: 8 dez. 2015.

BERNARDES, S. S.; TURINI, C. A.; MATSUO, T. Perfil das tentativas de suicídio por sobredose intencional de medicamentos atendidas por um centro de controle de intoxicações do Paraná, Brasil. Cadernos de Saúde Pública, Rio de Janeiro, v. 26, n. 7, p. 1.366-1.372, jul. 2010. Disponível em: <http://www.scielo.br/scielo.php?pid=s0102-311x2010000700015\&script=sci_arttext>. Acesso em: 30 nov. 2015.

BORGES, V. R.; WERLANG, B. S. G. Estudo de ideação suicida em adolescentes de 15 a 19 anos. Estudos de Psicologia, Natal, v. 11, n. 3, p. 345-351, 2006. Disponível em: <http://www.scielo.br/pdf/epsic/v11n3/12.pdf>. Acesso em: 11 dez. 2015. 
Félix; Tamires A. - Oliveira; Eliany Nazaré - Lopes; Marcos Venicios de Oliveira - Parente; José Reginaldo Feijão - Dias; Maria Socorro de Araújo - Moreira; Roberta Magda Martins BOTEGA, N. J. et al. Suicidal behavior in the community: prevalence and factors associated with suicidal ideation. Rev Bras Psiquiatr, v. 27, n. 1, p. 45-53, mar. 2005.

BRASIL. Portaria n. 1.876, de 14 de agosto de 2006. Diretrizes Nacionais para Prevenção do Suicídio. Brasília, 2006.

Projeto de Lei $n^{o}$ 498/2007. Estabelece a obrigatoriedade dos hospitais públicos e privados a notificarem os casos de atendimento a pessoas com diagnóstico de tentativa de suicídio. Brasília, 2007.

COÊLHO, B. M. et al. The influence of the comorbidity between depression and alcohol use disorder on suicidal behaviors in the São Paulo Epidemiologic Catchment Area Study, Brazil. Revista Brasileira de Psiquiatria, São Paulo, v. 32, n. 4, p. 396-408, abr. 2010. Disponível em: <http://www.scielo.br/scielo.php?script=sci_arttext\&pid=S1516-44462010000400013\&lng=en\&nrm=iso\&tlng=en> . Acesso em: 9 dez. 2015.

DIEHL, A.; LARANJEIRA, R. Suicide attempts and substance use in an emergency room sample. Jornal Brasileiro de Psiquiatria, Rio de Janeiro, v. 58, n. 2, p. 86-91, 2009. Disponível em: <http://www.scielo.br/pdf/jbpsiq/v58n2/v58n2a03.pdf>. Acesso em: 10 dez. 2015.

FERREIRA, M. H. F. et al. Suicide risk among inpatients at a university general hospital. Revista Brasileira de Psquiatria, v. 29, n. 1, p. 51- 54, 2007. Disponível em: <http://dx.doi.org/10.1590/S1516-44462006005000031>. Acesso em: 11 dez. 2015.

FICHER, A. M. F. T.; VANSAN, G. A. Tentativas de suicídio em jovens: aspectos epidemiológicos dos casos atendidos no setor de urgências psiquiátricas de um hospital geral universitário entre 1988 e 2004. Estudos de Psicologia, Campinas, v. 25, n. 3, p. 361-374, 2008. Disponível em: <http://www.scielo.br/pdf/estpsi/v25n3/a05v25n3.pdf>. Acesso em: 11 dez. 2015.

FIGEL, F. C.; MENEGATTIL, C. L.; PINHEIRO, E. P. N. Suicide attempts: a contingency analysis. Estudos de Psicologia, Campinas, v. 30, n. 2, p. 211-218, abr./ jun. 2013. Disponível em: <http://www.scielo.br/pdf/estpsi/v30n2/07.pdf >. Acesso em: 7 dez. 2015.

FONSECA, D. L. et al. Apoio social, eventos estressantes e depressão em casos de tentativa de suicídio: um estudo de caso-controle realizado em um hospital de emergência do Rio de Janeiro. Cadernos de Saúde Coletiva, Rio de Janeiro, v. 18, n. 2, p. 217- 228, 2010. Disponível em: <http://www.cadernos.iesc.ufrj.br/cadernos/images/csc/2010_2/artigos/CSCv18n2_217-228.pdf.>. Acesso em: 9 dez. 2015.

GAZALLE, F. K. et al. Polypharmacy and suicide attempts in bipolar disorder. Revista Brasileira de Psiquiatria, v. 29, n. 1, p. 35-38, ago. 2007. Disponível em: <http://www.scielo.br/pdf/rbp/nahead/2375.pdf>. Acesso em: 11 dez. 2015.

LIMA, D. D. et al. Tentativa de suicídio entre pacientes com uso nocivo de bebidas alcoólicas internados em hospital geral. Jornal Brasileiro de Psiquiatria, Rio de Janeiro, v. 59, n. 3, p. 167-172, jul. $2010 . \quad$ Disponível em: <http://www.scielo.br/pdf/jbpsiq/v59n3/a01v59n3.pdf>. Acesso em: 13 dez. 2015.

MEYER, A. et al. Mood disorders hospitalizations, suicide attempts, and suicide mortality among agricultural workers and residents in an area with intensive use of pesticides in Brazil. J Toxicol Environ Health A, v. 73, n. 13-14, p. 866-877, 2010.

NEVES, F. S. et al. Bipolar disorder first episode and suicidal behavior: are there differences according to type of suicide attempt? Revista Brasileira de Psiquiatria, São Paulo, v. 31, n. 2, p. 114-118, jun. $2009 . \quad$ Disponível em: <http://www.scielo.br/pdf/rbp/v31n2/v31n2a06.pdf>. Acesso em: 4 dez. 2015.

OLIVEIRA, M. I. V.; BEZERRA FILHO, J. G.; FEITOSA, R. F. G. Tentativas de suicídio atendidas em unidades públicas de saúde de Fortaleza-Ceará, Brasil. Revista de Salud Pública, v. 16, n. 5, p. 687-699, set./out. $2014 . \quad$ Disponível em: <http://www.scielosp.org/pdf/rsap/v16n5/v16n5a04.pdf>. Acesso em: 10 dez. 2015.

PIRES, D. X.; CALDAS, E. D.; RECENA, M. C. Uso de agrotóxicos e suicídios no Estado do Mato Grosso do Sul, Brasil. Cad Saúde Pública, v. 21, n. 2, p. 598-605, mar./abr. 2005. 
PIRES, M. C. C. et al. Risk factors of suicide attempts by poisoning: review. Trends Psychiatry Psychother, v. 36, n. 2, p. 63-74, 2014. Disponível em: <http://www.scielo.br/pdf/trends/v36n2/2237-6089-trends-36-02-00063.pdf> Acesso em: 6 dez. 2015.

Stressors in attempted suicide by poisoning: a sex comparison. Trends Psychiatry Psychother, Porto Alegre, v. 34, n. 1, p. 2530, 2012. Disponível em: 〈http://www.scielo.br/pdf/trends/v34n1/a06v34n1.pdf>. Acesso em: 10 dez. 2015.

QUARANTINI, L. C. et al. The impact of comorbid posttraumatic stress disorder on bipolar disorder patients. $J$ Affect Disord, v. 123, n. 1-3, p. 71-76, jun. 2010.

SANTOS, S. A. et al. Prevalência de transtornos mentais nas tentativas de suicídio em um hospital de emergência no Rio de Janeiro, Brasil. Cadernos de Saúde Pública, Rio de Janeiro, v. 25, n. 9, p. 2.064-2.074, set. 2009. Disponível em: <http://www.scielo.br/pdf/csp/v25n9/20.pdf>. Acesso em: 10 dez. 2015.

SILVA, V. F. et al. Fatores associados à ideação suicida na comunidade: um estudo de caso-controle. Cad Saúde Pública, v. 22, n. 9 , p. $1.835-1.843$, set. 2006.

SOUZA, M. T.; SILVA, M. D.; CARVALHO, R. Revisão integrativa: o que é e como fazer. Revista Einstein, São Paulo, v. 8, n. 1, p. 102-106, 2010. Disponível em: <http://www.astresmetodologias.com/material/O_que_e_RIL.pdf>. Acesso em: 19 nov. 2015

SOUZA, V. S. et al. Tentativas de suicídio e mortalidade por suicídio em um município no interior da Bahia. Jornal Brasileiro de Psiquiatria, Rio de Janeiro, v. 60, n. 4, p. 294-300, 2011. Disponível em: 〈http://www.scielo.br/pdf/jbpsiq/v60n4/a10v60n4.pdf>. Acesso em: 10 dez. 2015.

STEFANELLO, S. et al. Gender differences in suicide attempts: preliminary results of the multisite intervention study on suicidal behavior (SUPRE-MISS) from Campinas, Brazil. Revista Brasileira de Psiquiatria, São Paulo, v. 30, n. 2, p. 139-143, jun. 2008. Disponível em: <http://www.scielo.br/pdf/rbp/v30n2/2566.pdf>. Acesso em: 9 dez. 2015.

TARANTINO, M. Suicídio cresce no Brasil: mas isso poderia ser evitado. 2007. Disponível em: http://revistaplaneta.terra.com.br/secao/saude/suicidio-cresce-no-brasil

VIDAL, C. E. L.; GONTIJO, E. D. Tentativas de suicídio e o acolhimento nos serviços de urgência: a percepção de quem tenta. Caderno Saúde Coletiva, Rio de Janeiro, v. 21, n. 2, p. 108-114, 2013. Disponível em: $<$ http://www.scielo.br/pdf/cadsc/v21n2/02.pdf>. Acesso em: 10 dez. 2015.

WERNECK, G. L. et al. Tentativas de suicídio em um hospital geral no Rio de Janeiro, Brasil. Cadernos de Saúde Pública, Rio de Janeiro, v. 22, n. 10, p. 2.201-2.206, out. 2006. Disponível em: 〈http://www.scielo.br/pdf/csp/v22n10/19.pdf>. Acesso em: 9 dez. 2015.

WORLD HEALTH ORGANIZATION (WHO). Preventing suicide: a global imperative. Geneva, 2014. 